\title{
Time-resolved electron beam phase space tomography at a soft x-ray free-electron laser
}

\author{
Michael Röhrs, Christopher Gerth, Holger Schlarb, Bernhard Schmidt, and Peter Schmüser \\ Deutsches Elektronen-Synchrotron DESY, Notkestrasse 85, 22607 Hamburg, Germany
}

(Received 12 December 2008; published 19 May 2009)

\begin{abstract}
High-gain free-electron lasers (FELs) in the ultraviolet and x-ray regime put stringent demands on the peak current, transverse emittance, and energy spread of the driving electron beam. At the soft x-ray FEL FLASH, a transverse deflecting microwave structure (TDS) has been installed to determine these parameters for the longitudinally compressed bunches, which are characterized by a narrow leading peak of high charge density and a long tail. The rapidly varying electromagnetic field in the TDS deflects the electrons vertically and transforms the time profile into a streak on an observation screen. The bunch current profile was measured single shot with an unprecedented resolution of $27 \mathrm{fs}$ under FEL operating conditions. A precise single-shot measurement of the energy distribution along a bunch was accomplished by using the TDS in combination with an energy spectrometer. Variation of quadrupole strengths allowed for a determination of the horizontal emittance as a function of the longitudinal position within a bunch, the so-called slice emittance. In the bunch tail, a normalized slice emittance of about $2 \mu \mathrm{m}$ was found, in agreement with expectations. In the leading spike, however, surprisingly large emittance values were observed, in apparent contradiction with the low emittance deduced from the measured FEL gain. By applying three-dimensional phase space tomography, we were able to show that the bunch head contains a central core of low emittance and high local current density, which is presumably the lasing part of the bunch.
\end{abstract}

DOI: 10.1103/PhysRevSTAB.12.050704

PACS numbers: 41.60.Cr, 41.75.Ht, 41.85.Ew

\section{INTRODUCTION}

The requirements placed on peak current, energy spread, and transverse emittance of the driving electron beam in short-wavelength free-electron lasers (FELs) are extremely demanding. They can currently only be met by linear accelerators (linacs) equipped with high-brightness and low-emittance electron sources. The electron beam is subject to severe perturbations during its passage through a long linac and the magnetic chicanes used for longitudinal bunch compression. Accurate diagnostic tools for probing the electron beam are, therefore, essential for operating and optimizing the FEL. In contrast to storage rings, where the particle beam reaches an equilibrium state after many turns, significant fluctuations of beam parameters happen from shot to shot in linacs and necessitate diagnostic devices with single-shot capability. At the ultraviolet and soft $x$-ray FEL facility FLASH (free-electron laser in Hamburg), the requirements for lasing are presently fulfilled only within a short leading spike of the electron bunch, comprising some 10\%-20\% of the total bunch charge. Probing this lasing bunch section requires a time resolution on the femtosecond scale and makes accurate beam diagnostics a challenging task.

Very powerful diagnostic instruments are transverse deflecting microwave structures. A fast-deflector cavity was used for electron beam diagnostics at the Los Alamos freeelectron laser [1]. Traveling wave structures were developed at SLAC in the 1960s for the purpose of particle separation in secondary beams [2]. The usage for electron beam diagnostics was proposed in Ref. [3], a successful application during the commissioning of the injector of the Linac Coherent Light Source (LCLS) has been reported in [4]. The working principle of a transverse deflecting structure (TDS) is comparable to that of an ultrafast oscilloscope. A high-frequency electromagnetic field exerts a time-dependent transverse force on the electrons, analogous to the sawtooth voltage in the oscilloscope, and converts the temporal profile of the bunch into a transverse (here vertical) streak on an observation screen. The bunch charge density profile can thus be measured single shot. Furthermore, appropriate variations of quadrupole strengths in the beam line upstream of the TDS allow for time-resolved horizontal phase space tomography. A crucial quantity that can be deduced from such a measurement is the horizontal slice emittance. A second screen mounted behind a dipole magnet is utilized to measure the energy distribution along the bunch axis and to carry out a longitudinal phase space analysis.

In terms of time resolution, the TDS is superior to any other time-domain method currently used for longitudinal bunch diagnostics. Because of its outstanding performance and versatility, a TDS is foreseen in the driving accelerator of many current FEL projects. In this paper, we report on the application of the TDS for high-resolution beam characterization at the FLASH facility.

The paper is organized as follows. The FLASH facility and the experimental setup are described in Sec. II. Section III deals with the experimental procedures and the data analysis. Experimental results obtained with an 
uncompressed beam and under FEL operating conditions are presented in Secs. IV and V, respectively. The results are summarized in Sec. VI.

\section{EXPERIMENTAL SETUP}

\section{A. The free-electron laser in Hamburg}

The free-electron laser FLASH is a user facility providing radiation at a fundamental wavelength between 6.5 and $50 \mathrm{~nm}$. It is an upgrade of the vacuum ultraviolet (VUV) FEL at the TESLA Test Facility (TTF), which served as a test bed for the superconducting cavities developed for the TESLA Linear Collider Project [5]. At an early stage, the TTF facility was augmented with an undulator to produce FEL radiation, relying on the principle of self-amplified spontaneous emission (SASE). In 2001, the VUV-FEL was the first FEL to achieve lasing and saturation at wavelengths below $100 \mathrm{~nm}[6,7]$. After upgrades in energy by additional acceleration modules and an increase in undulator length, lasing has been achieved down to $13 \mathrm{~nm}$ in 2006 [8] and $6.5 \mathrm{~nm}$ in 2007 [9]. Aside from being a user facility, FLASH serves as a pilot project for the planned European x-ray FEL [10] and as a test bed for dedicated studies related to the International Linear Collider project [11].

A schematic of FLASH is shown in Fig. 1. The electron source is a laser-driven photocathode mounted in a $1.3 \mathrm{GHz}$ copper cavity [12]. The linac consists of six acceleration modules (ACC1 to ACC6), each equipped with eight $1.3 \mathrm{GHz}$ superconducting cavities [13] and a quadrupole doublet, and two magnetic bunch compressors $\mathrm{BC} 1$ and $\mathrm{BC}$. The radio frequency (rf) gun typically generates trains of electron bunches at a rate of $5 \mathrm{~Hz}$, each train comprising up to 800 bunches with a time spacing of $1 \mu \mathrm{s}$. The electron bunches leaving the gun have a charge of 0.5 to $1 \mathrm{nC}$ and an energy of about
$5 \mathrm{MeV}$. The energy is boosted to $130 \mathrm{MeV}$ in module ACC1. The rf cavities are operated off-crest in order to induce an energy gradient along the bunch axis that is needed for longitudinal bunch compression in the magnetic chicane $\mathrm{BC} 1$. Modules ACC $2 / 3$ accelerate the particles to about $380 \mathrm{MeV}$, and a second compression takes place in BC2. The acceleration modules ACC4 to ACC6 boost the energy up to a maximum of $1 \mathrm{GeV}$.

The undulator magnet consists of six $4.5 \mathrm{~m}$ long segments. Two collimators in combination with a double-bend magnetic deflection (a so-called "dog leg") protect the permanent magnets of the undulator against radiation damage by beam halo. The total length of the facility, including the hall for FEL user experiments, is about $300 \mathrm{~m}$.

\section{B. The diagnostic beam line}

The transverse deflecting structure is installed downstream of the acceleration modules (Fig. 1). Two optical transition radiation (OTR) stations, labeled OTR1 and OTR2, are placed $9.5 \mathrm{~m}$, respectively $24.5 \mathrm{~m}$, downstream of the TDS. The screen of station OTR1 is mounted with a horizontal offset of $7 \mathrm{~mm}$ between the edge of the screen and the beam axis and is used in combination with a stripline kicker magnet located $1.5 \mathrm{~m}$ upstream of the TDS. The kicker magnet is driven with fast current pulses of $1.5 \mu$ s duration, so that a single bunch can be kicked out of a train with $1 \mu \mathrm{s}$ bunch spacing. The station OTR2 is mounted behind a horizontally deflecting bending magnet. Single-shot energy measurements require linac operation with only one bunch per train. Several quadrupole magnets, beam position monitors, and steerer magnets are installed between the second bunch compressor and the OTR screens and are utilized to tune the accelerator optics and the beam orbit.

The OTR screens are aluminum-coated silicon wafers. They are mounted inside the beam pipe and tilted by $45^{\circ}$

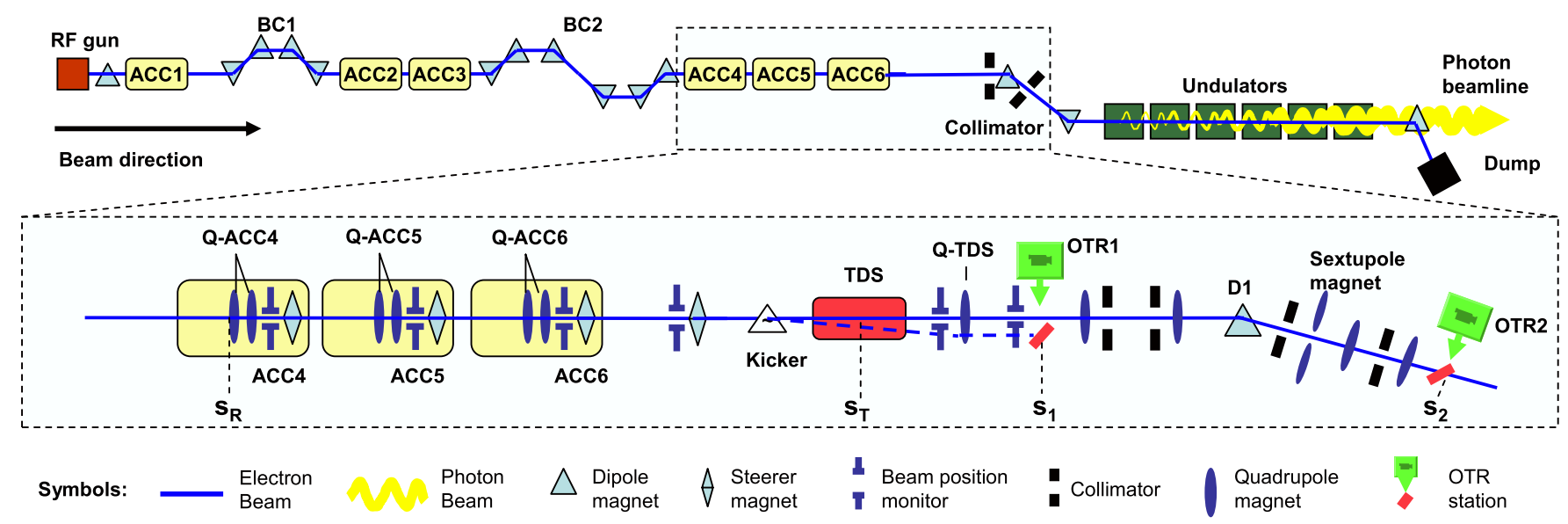

FIG. 1. (Color) Top: Schematic layout of FLASH (not to scale). Bottom: Zoom into the diagnostic beam line comprising the transverse deflecting structure (TDS) and two optical transition radiation stations OTR1 and OTR2. Only a selection of the beam position monitors, steering magnets, and OTR stations installed in this beam line is shown. Also indicated are the longitudinal positions $s_{R}$ (phase space reconstruction point), $s_{T}$ (center of TDS), $s_{1}$ (screen OTR1), and $s_{2}$ (screen OTR2). 
TABLE I. Properties of the stations OTR1 and OTR2 [14,15]. Abbreviations: horizontal (h), vertical (v).

\begin{tabular}{lcc}
\hline \hline Station & OTR1 & OTR2 \\
\hline Screen size $[\mathrm{mm}]$ & $8(\mathrm{~h}) \times 26.5(\mathrm{v})$ & $30 \times 30$ \\
Camera & Basler A311f & Basler A301f \\
Video output format $[\mathrm{bit}]$ & $8 / 12$ used: 8 & 8 \\
Number of pixels & $480(\mathrm{~h}) \times 640(\mathrm{v})$ & $640(\mathrm{~h}) \times 480(\mathrm{v})$ \\
Pixel width $[\mu \mathrm{m}]$ & 9.9 & 9.9 \\
Exposure time $[\mu \mathrm{s}]$ & $\geq 100$ & $\geq 20$ \\
Focal length $f[\mathrm{~mm}]$ & 200 & 200 \\
Image scale $[\mu \mathrm{m} / \mathrm{pixel}]$ & $27(\mathrm{~h}) / 24(\mathrm{v})$ & 25 \\
rms resolution $[\mu \mathrm{m}]$ & $\lesssim 20$ & $\lesssim 20$ \\
\hline \hline
\end{tabular}

with respect to the electron beam axis. The OTR light leaves the vacuum chamber through silica windows and is imaged by CCD cameras, which are triggered in synchronism with the bunched electron beam. Selected properties of both stations are listed in Table I.

The TDS is a disk-loaded traveling wave structure of constant impedance, operated at a frequency $f_{0}=$ $2.86 \mathrm{GHz}$ in a hybrid mode with transverse electric and magnetic fields. It was installed at the FLASH linac in a collaboration between SLAC and DESY $[14,16]$. The TDS is powered with rf pulses of $1 \mu \mathrm{s}$ duration. The short filling time of $0.64 \mu$ s allows the selection of a single bunch out of a train with $1 \mu \mathrm{s}$ bunch spacing. The nominal input power of the structure is $20 \mathrm{MW}$. Because of space limitations, the TDS is driven by a remote 44 MW klystron through a $75 \mathrm{~m}$ long waveguide (2.6 dB attenuation). The rf input power and phase can be remotely controlled. The structure and the waveguide are temperature stabilized at $(45 \pm 0.1)^{\circ} \mathrm{C}$ by a water-cooling system. The temperature was chosen such that the resonance frequency of the TDS is $11 / 5$ of the linac of of $1.3 \mathrm{GHz}$. A dedicated synchronization circuit allows for operation of the TDS synchronously with the bunched electron beam with a timing jitter of $145 \mathrm{fs}(\mathrm{rms})$ [16]. The parameters of the TDS setup are summarized in Table II.

An electron passing the TDS along the symmetry axis experiences a microwave field with a vertical electric and a horizontal magnetic component. The rapidly varying vertical Lorentz force has the same magnitude over the entire aperture and thus provides an aberration-free deflection of

TABLE II. Properties of the TDS at FLASH $[2,14]$.

\begin{tabular}{lc}
\hline \hline Length & $3.64 \mathrm{~m}$ \\
Cell length & $35 \mathrm{~mm}$ \\
Iris aperture & $44.88 \mathrm{~mm}$ \\
Disk thickness & $5.84 \mathrm{~mm}$ \\
Nominal frequency & $2.856 \mathrm{GHz}$ \\
Phase shift per cell & $2 / 3 \pi$ \\
Nominal input power & $20 \mathrm{MW}$ \\
Filling time & $0.64 \mu \mathrm{s}$ \\
\hline \hline
\end{tabular}

particles moving parallel to the axis. Details on the electromagnetic field pattern in the structure can be found in $[2,3]$.

The deflection angle $\Delta y^{\prime}$ imposed on an electron of momentum $p$ is approximately given by [3]

$$
\Delta y^{\prime} \approx \frac{e V_{0}}{c|p|} \sin \psi,
$$

where $\psi$ is the rf phase and $V_{0}$ is the peak deflecting voltage, which is related to the input power $P$ via the empirical relation

$$
V_{0}[\mathrm{MV}] \approx 5.824 \sqrt{P[\mathrm{MW}]} .
$$

\section{EXPERIMENTAL PROCEDURES}

Because of the rapid time variation of the rf field, the deflecting force acting on an electron depends sensitively on its longitudinal position $\zeta$ inside the bunch, defined as the distance from the center of mass of the bunch. The deflection angle $\Delta y^{\prime}(\zeta)$ is given by

$$
\Delta y^{\prime}(\zeta)=\frac{e V_{0}}{c|p|} \sin \left(2 \pi f_{0} \zeta / c+\psi_{c}\right),
$$

where $p$ is the particle momentum and $\psi_{c}$ the rf phase with respect to the center of mass of the bunch. For optimum time resolution, the structure is operated at zero crossing of the transverse force $\left(\psi_{c}:=0\right)$, hence Eq. (2) reduces to

$$
\Delta y^{\prime}(\zeta)=\frac{e V_{0}}{c|p|} \sin \left(2 \pi f_{0} \zeta / c\right) \approx \frac{2 \pi f_{0} e V_{0}}{c^{2}|p|} \zeta .
$$

The action of the TDS can in good approximation be described by a localized kick at the center $s=s_{T}$. We call $M^{y} \equiv M^{y}\left(s_{1}, s_{T}\right)$ the vertical transfer matrix from the center of the TDS at $s_{T}$ to the OTR1 screen at $s=s_{1}$. When the TDS is switched off, the vertical particle coordinates are transformed as

$$
\left[\begin{array}{c}
y\left(s_{1}\right) \\
y^{\prime}\left(s_{1}\right)
\end{array}\right]=M^{y}\left[\begin{array}{c}
y\left(s_{T}\right) \\
y^{\prime}\left(s_{T}\right)
\end{array}\right] .
$$

With the TDS in operation, the particle position on the OTR screen receives a $\zeta$-dependent vertical shift

$$
\Delta y(\zeta)=M_{1,2}^{y} \Delta y^{\prime}(\zeta) \approx S \zeta,
$$

where we have defined the shear parameter $S$ by

$$
S=M_{1,2}^{y} \frac{2 \pi f_{0} e V_{0}}{c^{2}|p|} .
$$

The above equations describe a spatial shearing. If the shear parameter $S$ is very large, the observed charge density profile on the OTR screen will provide a faithful image of the bunch time profile. Usually, however, the nonvanishing vertical beam size leads to a smearing of the profile. 


\section{A. Time profile of the beam current}

In order to deduce the temporal profile of the beam current from the light intensity profile observed on the screen OTR1, the shear parameter $S$ must be known. It can be determined by varying the rf phase of the TDS around the zero-crossing phase and measuring the resulting vertical shift of the beam on the OTR screen. Such a calibration is shown in Fig. 2. Good linearity is observed, the slope yielding the shear parameter $S$. The accuracy is limited by a timing jitter of about $145 \mathrm{fs}$ (rms) between the beam and the rf input power which leads to fluctuations of the vertical beam position on the screen of roughly $40 \mu \mathrm{m}$ for moderate input power $(S \simeq 1)$ and up to $860 \mu \mathrm{m}$ for high input power $(S \simeq 20)$. For the measurements presented in this paper, the resulting rms error of the shear parameter is in the range from $1 \%$ for uncompressed bunches to $12 \%$ under FEL operating conditions.

As an example for a measurement with large shear parameter, we show in Fig. 3 the longitudinal profile of a compressed electron bunch, typical for FEL operation. The intensity scale of the CCD image can be translated into a charge density scale by measuring the total bunch charge with a toroid and equating it to the integrated intensity of the CCD image. A prerequisite of this calibration, and of OTR-based beam diagnostics in general, is that the light intensity scales linearly with the bunch charge. It is important to rule out contributions from coherent optical transition radiation (COTR) as they would disturb the linearity and could have a particularly detrimental effect on the high-intensity region in the bunch head.

Strong light intensities in the visible range were observed during the commissioning phase of the Linac Coherent Light Source at SLAC, and these were attributed to COTR [17]. Using accelerator settings that were very different from normal FEL operation conditions, some evidence for COTR could also be established at FLASH [18]. In these measurements large shot-to-shot fluctuations of the light intensity were observed.

The arguments against a significant COTR contribution to our CCD images are as follows. (i) The majority of our
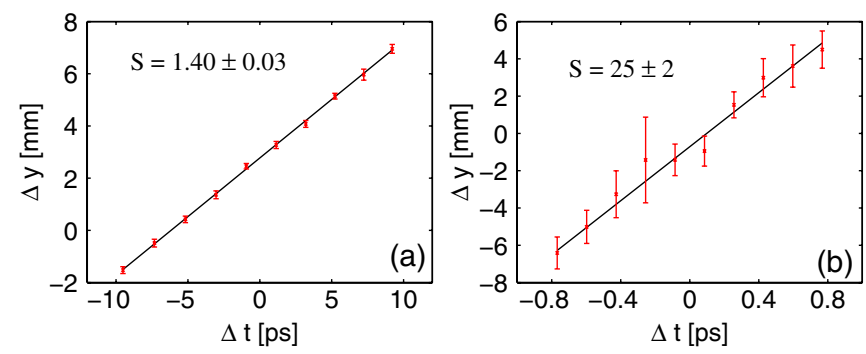

FIG. 2. (Color) Vertical beam position on the screen OTR1 as a function of $\Delta t$, the time delay between bunch arrival and zerocrossing phase of the TDS. (a) Low rf power, the shear parameter derived from this calibration is $S=1.40 \pm 0.03$; (b) high rf power, $S=25 \pm 2$.
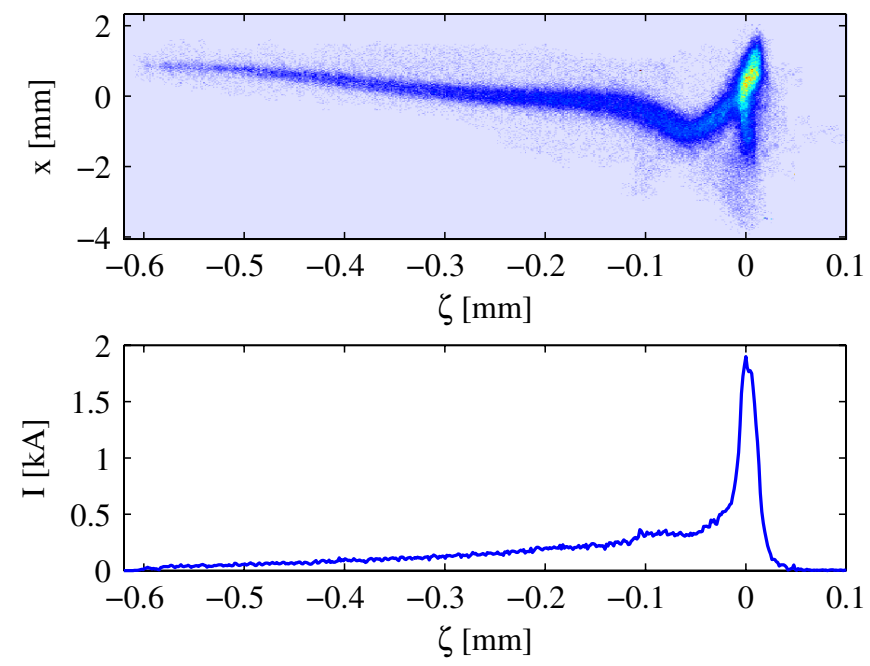

FIG. 3. (Color) Top: Two-dimensional CCD image of a single compressed electron bunch whose longitudinal charge density profile is translated into a spatial distribution on an OTR screen. The bunch head at the right side. Bottom: Current as a function of the internal bunch coordinate $\zeta$. The peak current is $1.8 \mathrm{kA}$ with an estimated uncertainty of $10 \%$.

TDS measurements were carried out with machine settings close to FEL operation, and during the course of the experiment no indication of excessive fluctuations was seen. (ii) A comparative study of the time profile of compressed bunches was carried out using the TDS and a highresolution electro-optic (EO) detector [19]. The EO crystal was mounted inside the electron beam pipe and recorded the transient electric field of the passing bunch and thereby its longitudinal charge density. Excellent agreement in the shape of the leading spike was obtained. (iii) In Fig. 4 we depict the shot-to-shot correlation between the integrated intensity of the CCD images, recorded under FEL operating conditions, and the corresponding total bunch charges $Q$ as measured by a toroid. The light intensity scales linearly with $Q$, and a good fit is obtained by the straight lines which all pass through the origin. (iv) In recent spectroscopic measurements at the coherent transition radiation (CTR) beam line [20] at FLASH, coherent radiation in the near infrared was detected [21]. The CTR intensity, however, exhibited a steep drop towards the visible range. The coherent intensity in the wavelength range from 3 to $10 \mu \mathrm{m}$ was found to be almost the same for uncompressed and compressed bunches. Removing the leading spike from the compressed bunches by means of an energy collimator in the bunch compressor chicane had almost no effect on the 3 to $10 \mu \mathrm{m}$ radiation but led to a reduced intensity in the $20-40 \mu \mathrm{m}$ range. From this observation we conclude that the short-wavelength CTR originates mainly from the uncompressed section of the bunch and not from the high-current region in the leading spike. In none of the TDS measurements reported here did we find any evidence for coherent optical light in the bunch 


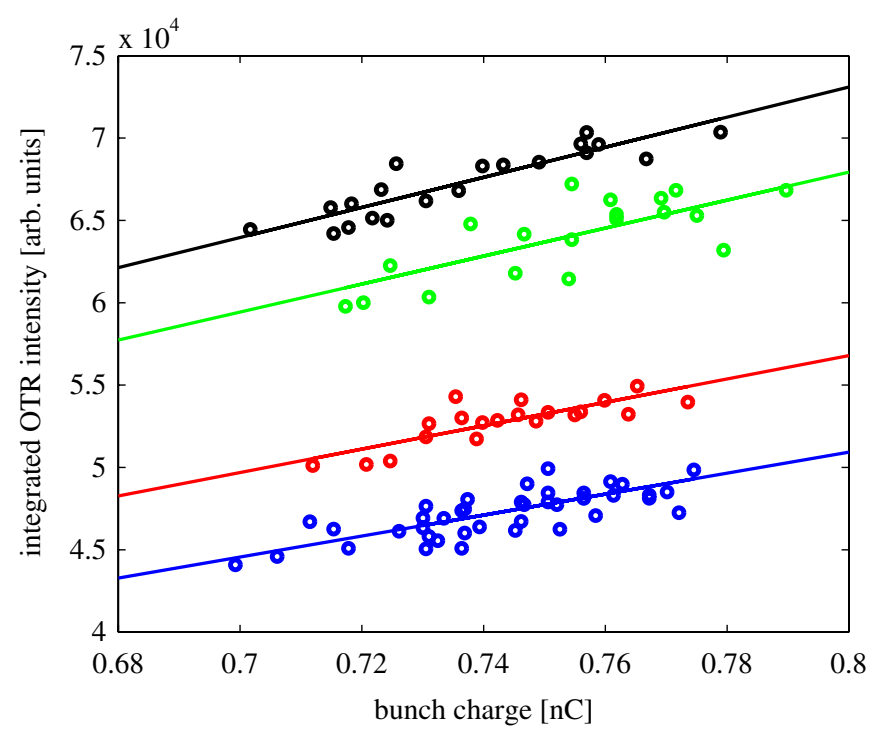

FIG. 4. (Color) Shot-to-shot fluctuations of the integrated OTR intensity at the screen OTR1 and of the bunch charge under FEL operating conditions $(E=494 \mathrm{MeV})$. The different colors refer to different gains of the CCD camera which were chosen to avoid CCD saturation. The straight lines pass through the origin.

tail. Then, from the observations in [21], it appears rather unlikely that the bunch head should be seriously affected by COTR.

Combining the above observations, we conclude that a possible COTR contribution to the bunch head region, if it exists at all, is insignificant in comparison with the estimated $10 \%$ uncertainty of the peak current determination by the TDS setup.

We investigate now the influence of a finite vertical beam emittance on the longitudinal resolution of a measured current profile. When the TDS is switched off, the rms beam size at the screen OTR 1 at $s_{1}$ is

$$
\sigma_{y}^{\text {off }}\left(s_{1}\right)=\sqrt{\varepsilon_{y} \beta_{y}\left(s_{1}\right)}
$$

The transfer matrix can be written in the form

$$
M_{1,2}^{y}\left(s_{1}, s_{T}\right)=\sqrt{\beta_{y}\left(s_{1}\right) \beta_{y}\left(s_{T}\right)} \sin \left(\Delta \phi_{y}\right),
$$

where $\beta_{y}\left(s_{T}\right), \beta_{y}\left(s_{1}\right)$ is the vertical beta function at the center of the TDS and the OTR screen, respectively, and

$$
\Delta \phi_{y}=\int_{s_{T}}^{s_{1}} \frac{1}{\beta_{y}(s)} d s
$$

is the phase advance between the TDS and the OTR1 screen. Using Eqs. (6)-(8), one obtains for the longitudinal resolution

$$
\sigma^{\text {long }}=\frac{\sigma_{y}^{\text {off }}}{|S|}=\frac{\sqrt{\varepsilon_{y}}}{\sqrt{\beta_{y}\left(s_{T}\right)}\left|\sin \left(\Delta \phi_{y}\right)\right|} \frac{c^{2}|p|}{2 \pi f_{0} e V_{0}} .
$$

This equation shows that the resolution can be improved by increasing the beta function $\beta_{y}\left(s_{T}\right)$ in the center of the TDS, thereby decreasing the beam divergence, and by choosing a betatron phase advance $\Delta \phi_{y}$ such that $\left|\sin \left(\Delta \phi_{y}\right)\right| \approx 1$.

Since the TDS at FLASH was installed in an existing linac with a given magnet lattice, the beam optics could only be varied within certain limits. A design optics with $\beta_{y}\left(s_{T}\right)=36 \mathrm{~m}, \beta_{y}\left(s_{1}\right)=5 \mathrm{~m}$, and a phase advance $\Delta \phi_{y} \approx 63^{\circ}$ was found to be a good choice.

\section{B. Horizontal phase space}

A key parameter of the particle distribution in horizontal phase space is the rms transverse emittance

$$
\varepsilon_{x}=\sqrt{\operatorname{det}\left(\Sigma^{x}\right)}
$$

where $\Sigma^{x} \equiv \Sigma^{x}(s)$ denotes the covariance matrix of the beam (also called beam matrix):

$$
\begin{aligned}
\Sigma^{x}(s) & =\left[\begin{array}{cc}
\left\langle(x-\langle x\rangle)^{2}\right\rangle & \left\langle(x-\langle x\rangle)\left(x^{\prime}-\left\langle x^{\prime}\right\rangle\right)\right\rangle \\
\left\langle(x-\langle x\rangle)\left(x^{\prime}-\left\langle x^{\prime}\right\rangle\right)\right\rangle & \left\langle\left(x^{\prime}-\left\langle x^{\prime}\right\rangle\right)^{2}\right\rangle
\end{array}\right] \\
& \equiv \varepsilon_{x}\left[\begin{array}{cc}
\beta_{e}(s) & -\alpha_{e}(s) \\
-\alpha_{e}(s) & \gamma_{e}(s)
\end{array}\right] .
\end{aligned}
$$

Here, $x=x(s)$ is the horizontal offset from the design orbit, $x^{\prime}(s)=d x / d s$ is the slope, and $\beta_{e}(s), \alpha_{e}(s), \gamma_{e}(s)$ are the beam ellipse parameters. In a linear beam transport line without acceleration, the beam matrix transforms according to

$$
\Sigma^{x}\left(s_{1}\right)=M^{x}\left(s_{1}, s_{R}\right) \Sigma^{x}\left(s_{R}\right)\left[M^{x}\left(s_{1}, s_{R}\right)\right]^{t},
$$

where $\left[M^{x}\left(s_{1}, s_{R}\right)\right]^{t}$ is the transpose of $M^{x}\left(s_{1}, s_{R}\right)$. The emittance is conserved in this transformation, since $\operatorname{det}\left[M^{x}\left(s_{1}, s_{R}\right)\right]=1$.

The covariance matrix $\Sigma^{x}\left(s_{R}\right)$ at the reconstruction point $s=s_{R}$ (see Fig. 1) can be computed by measuring the position variance $\Sigma_{1,1}^{x}\left(s_{1}\right)=\left\langle\left[x\left(s_{1}\right)-\left\langle x\left(s_{1}\right)\right\rangle\right]^{2}\right\rangle$ for at least three different beam transfer matrices $M^{x}\left(s_{1}, s_{R}\right)$. In our experiments, more than three measurements were performed and a least squares method was applied to determine the covariance matrix and the emittance, following the procedure explained in [22]. The TDS permits the determination of the position variance as a function of $\zeta$. Hence, the beam ellipse parameters and the emittance can be computed for slices of the bunch. It is important to note that this technique implicitly relies on the constancy of the respective bunch properties from shot to shot.

From the horizontal intensity profiles obtained at different quadrupole settings, the horizontal phase space distribution can be reconstructed using an algorithm for computerized tomography $[23,24]$. We applied a maximum entropy algorithm, which minimizes reconstruction artifacts and provides reliable results $[25,26]$. Again, the TDS allows for the application of this technique to separate longitudinal bunch sections, thus providing a three- 
dimensional tomographic reconstruction of electron bunches. A similar approach using a magnetic spectrometer providing picosecond resolution is described in [27].

The optics has to be chosen carefully to obtain both a high longitudinal resolution and a good phase space reconstruction. The phase space reconstruction requires a variation of the horizontal betatron phase at the OTR screen of up to $180^{\circ}$. To preserve a good longitudinal resolution, several quadrupoles upstream of the TDS (QACC4 to Q-ACC6, see Fig. 1) were changed simultaneously in such a way that the vertical beta function at the TDS remained nearly constant [28]. In the slice emittance measurements, the horizontal betatron phase $\phi_{x}$ at the screen (station OTR1 in Fig. 1) was varied in 10 to 15 equidistant steps over an interval of $180^{\circ}$, and 20 to 30 images were taken at each step. The large number of steps allowed for reliable results without the necessity for precisely matching the beam to the design optics.

The emittance and phase space analysis is affected by error sources such as background signals in the CCD images, shot-to-shot fluctuations of the horizontal bunch profile, the finite resolution of the imaging system, and errors in beam energy and quadrupole strengths. The resulting rms emittance error is estimated to be $\leqslant 20 \%$ [28].

There is another class of error sources depending on specific beam properties such as space charge forces and dispersive effects resulting from beam energy spread. In order to study the accuracy that can be expected under FEL operating conditions, a so-called start-to-end simulation [29] of the FLASH accelerator was carried out in order to generate a known particle distribution in phase space resembling that of the real electron beam in FLASH. The effects of space charge forces and coherent synchrotron radiation were taken into account. The computed phase space distribution at the reconstruction point $s_{R}$ was used as an input for a simulation of the measurement process and the analysis procedures. The electrons were tracked from this point to the screen OTR1 at $s=s_{1}$ for all quadrupole settings used in a real beam measurement. The vertical streak applied by the TDS and the deflection by the kicker magnet were included in the tracking calculation. From the resulting particle distributions on the OTR1 screen, digital images were generated and subject to the same analysis procedures as the real data.

The tomographic reconstruction of the simulated horizontal phase space distribution in the bunch head region is shown in Fig. 5. If space charge forces are ignored in the particle tracking between $s_{R}$ and $s_{1}$, one obtains excellent agreement between the input distribution and the reconstructed phase space distribution, compare Figs. 5(a) and 5(b). This gives confidence that the analysis procedures are indeed correct. The inclusion of space charge forces, using the code ASTRA [30], leads to slight distortions [Fig. 5(c)]. The kicker magnet causes only minor additional distortions [Fig. 5(d)]. These distortions have
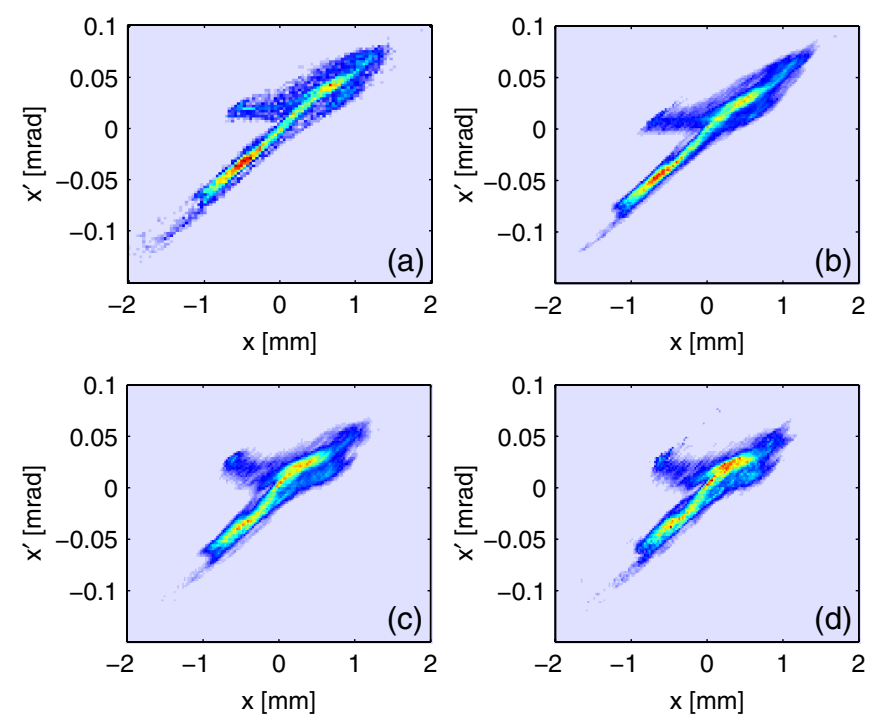

FIG. 5. (Color) Tomographic reconstruction of a simulated particle distribution. (a) Horizontal phase space of the bunch head at the reconstruction point $s=s_{R}$ as predicted by a start-to-end simulation of the FLASH linac under FEL operating conditions (beam energy $E=494 \mathrm{MeV}$, bunch charge $Q=0.72 \mathrm{nC}$ ). (b) Tomographic reconstruction of the distribution using only linear transfer matrices for particle tracking between the reconstruction point $s_{R}$ and the observation screen OTR1 at $s=s_{1}$. (c) Space charge forces are taken into account. (d) Space charge forces and the dispersive effects caused by the kicker are taken into account.

little influence on the reconstructed slice emittance which agrees very well with the slice emittance predicted by the start-to-end simulation (Fig. 6). In summary, we can state that our analysis procedures are well understood and yield reliable results.

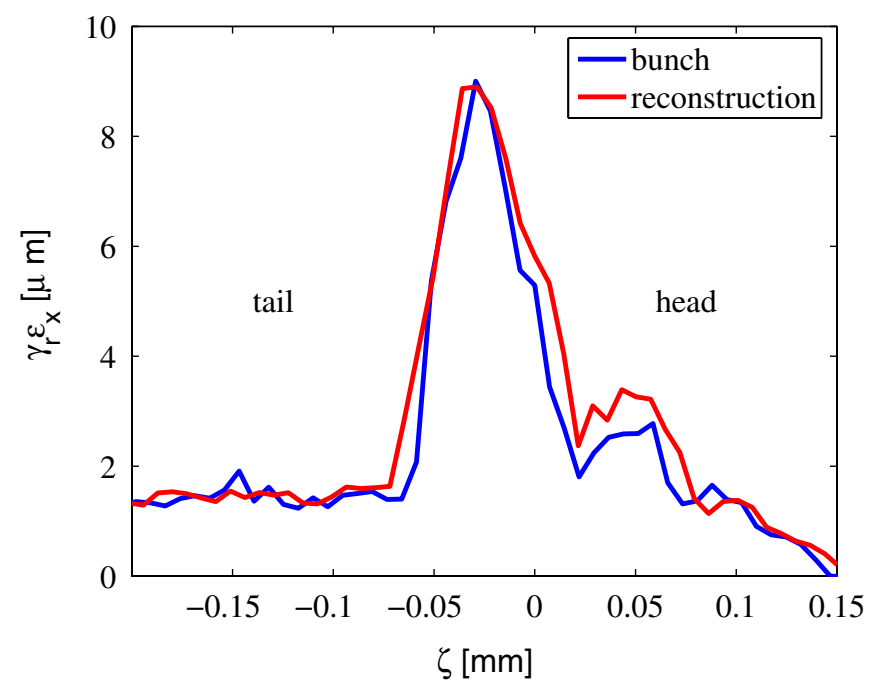

FIG. 6. (Color) Simulation of a slice emittance measurement. Blue curve: slice emittance as predicted by the start-to-end simulation of FLASH. Red curve: reconstructed slice emittance. 


\section{Longitudinal phase space}

The station OTR2 (position $s=s_{2}$ ) in Fig. 1 is located downstream of the horizontally deflecting dipole magnet D1 bending the beam by a nominal angle of $3.5^{\circ}$. A particle with relative momentum deviation $\delta=\Delta p / p$ will be observed with a horizontal displacement on the screen OTR2 given by

$$
x\left(s_{2}\right)=D_{x}\left(s_{2}\right) \delta,
$$

where $D_{x}(s)$ is the horizontal dispersion generated by the dipole. The horizontal position on the screen depends linearly on the momentum deviation. When the TDS is switched on, the OTR pattern provides therefore a direct image of the density distribution in longitudinal phase space $(\zeta, \delta)$.

The rms resolution $\sigma_{\delta}$ is

$$
\sigma_{\delta}=\frac{\sqrt{\varepsilon_{x} \beta_{x}\left(s_{2}\right)}}{D_{x}\left(s_{2}\right)} .
$$

The accelerator optics was optimized to simultaneously obtain a good longitudinal resolution according to Eq. (9) and a good momentum resolution, implying small $\beta_{x}\left(s_{2}\right)$ and large $D_{x}\left(s_{2}\right)$. Typical values are $\beta_{x}\left(s_{2}\right)=5 \mathrm{~m}$ and $D_{x}\left(s_{2}\right)=0.3 \mathrm{~m}$, resulting in $\sigma_{\delta}=3.4 \times 10^{-4}$ and $\sigma_{E}=$ $170 \mathrm{keV}$ for $E=500 \mathrm{MeV}$ and a normalized beam emittance of $2 \mu \mathrm{m}$.

\section{EXPERIMENTS WITH UNCOMPRESSED BUNCHES}

The beam dynamics in the linear accelerator of FLASH is adequately described by linear transport theory when all acceleration modules are operated on-crest. In this case the beam energy spread is minimal, and the bunches receive little compression in the magnetic chicanes. Collective effects such as coherent synchrotron radiation and space charge forces are therefore greatly reduced. These conditions are ideally suited to test the experimental methods. As a useful by-product, one gains information on the initial beam properties behind the electron gun, in particular, the bunch length and the slice emittance. In order to cover a large time window the TDS was operated with a small shear parameter $S=1$.

\section{A. Longitudinal phase space}

A typical longitudinal phase space distribution of an uncompressed bunch is presented in Fig. 7. The distribution shows the curvature imprinted on the bunch by the accelerating fields. The bunch center was accelerated oncrest and gained the most energy, while the particles in the leading and the trailing edge received a lower energy gain. The overall rms energy spread of the bunch is $585 \mathrm{keV}$ (average value from 30 bunches). The energy spread $\sigma_{E}(\zeta)$ as a function of the internal bunch coordinate $\zeta$ (slice energy spread) has its minimum value of $150 \mathrm{keV}$ in the

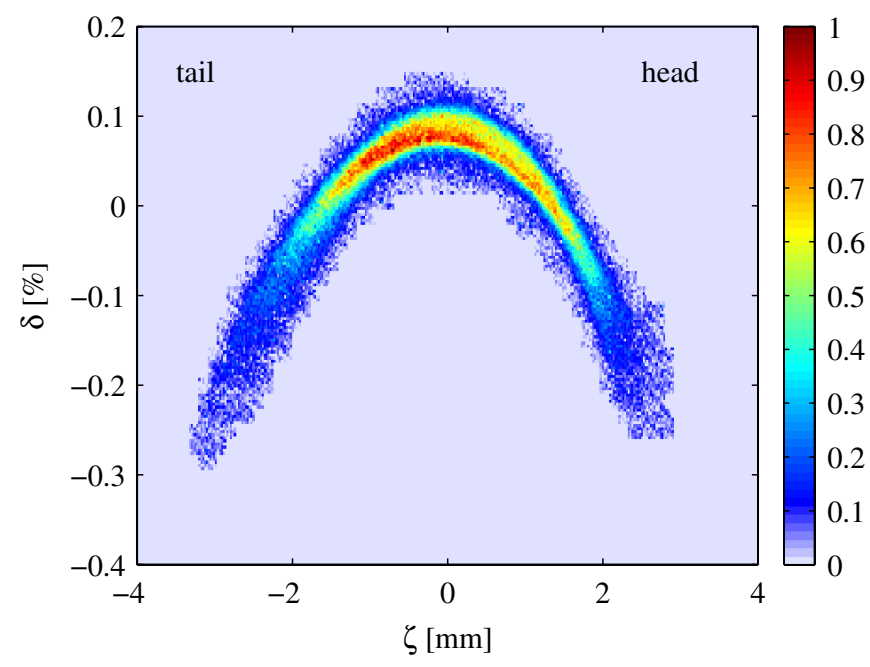

FIG. 7. (Color) Single-shot measurement of the particle density distribution of an uncompressed bunch in longitudinal phase space $(\zeta, \delta)(E=650 \mathrm{MeV}, Q=1 \mathrm{nC})$. The bunch compressor magnets were switched off.

center of the bunch. The true energy spread of the beam is far lower; in the injector section, it was measured to be less than $5 \mathrm{keV}$ [31]. The value of $150 \mathrm{keV}$, therefore, provides an estimate for the energy resolution of the experimental setup.

\section{B. Current profile}

The current profile of a typical uncompressed bunch is shown by the red curve in Fig. 8. In order to suppress any

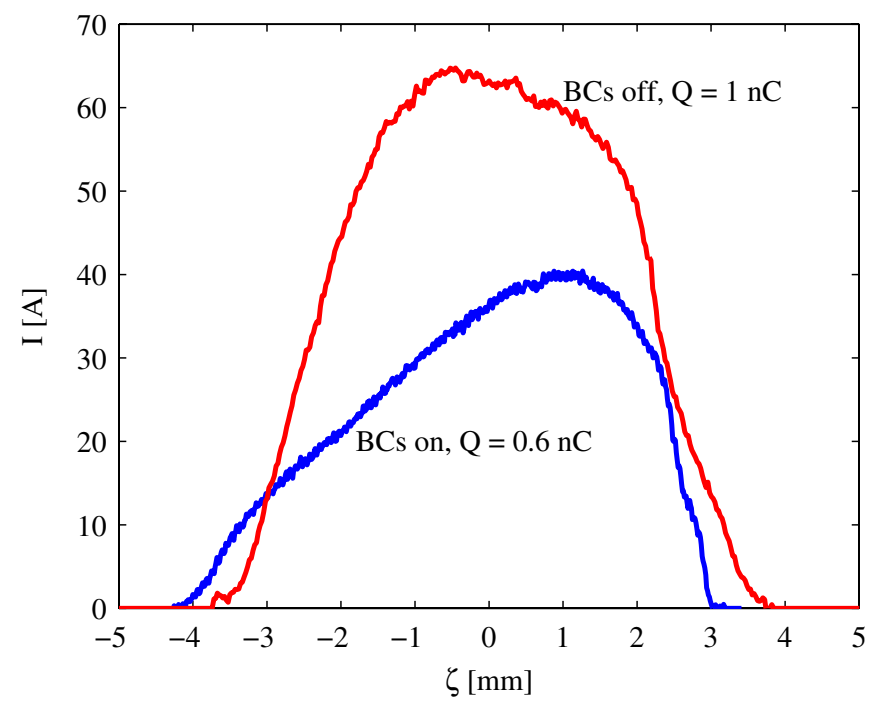

FIG. 8. (Color) Beam current $I$ as a function of the internal bunch coordinate $\zeta$ for on-crest operation of all acceleration modules. Red curve: dipoles of the magnetic chicanes switched off ( $E=650 \mathrm{MeV}, Q=1.0 \mathrm{nC}$ ). Blue curve: dipoles of the bunch compressor chicanes switched on $(E=490 \mathrm{MeV}, Q=$ $0.6 \mathrm{nC})$. 
compression effects, the bunch compressor magnets were switched off. The current profile is roughly symmetric with respect to the bunch center at $\zeta=0$. The measured peak current is $75 \mathrm{~A}$. The rms bunch length is $1.5 \mathrm{~mm}$. The total length over which a current was detectable is approximately $7 \mathrm{~mm}$.

When the BC magnets are switched on (blue curve), the profile acquires an asymmetry which is due to the fact that electrons in the head and tail region of the bunch are not accelerated on-crest, but on the slope of the cosine-shape rf wave. From Fig. 7 it is easy to understand that the head region will be compressed and the tail will be stretched in the chicanes. The peak current of $40 \mathrm{~A}$ is lower than in the previous case because of the smaller bunch charge of $0.6 \mathrm{nC}$.

\section{Horizontal phase space}

A typical result for the slice emittance of an uncompressed bunch is shown in Fig. 9. The results were obtained by determining the horizontal rms slice widths for each of the quadrupole settings, using the entire beam intensity contained in the slice. The normalized slice emittance is in
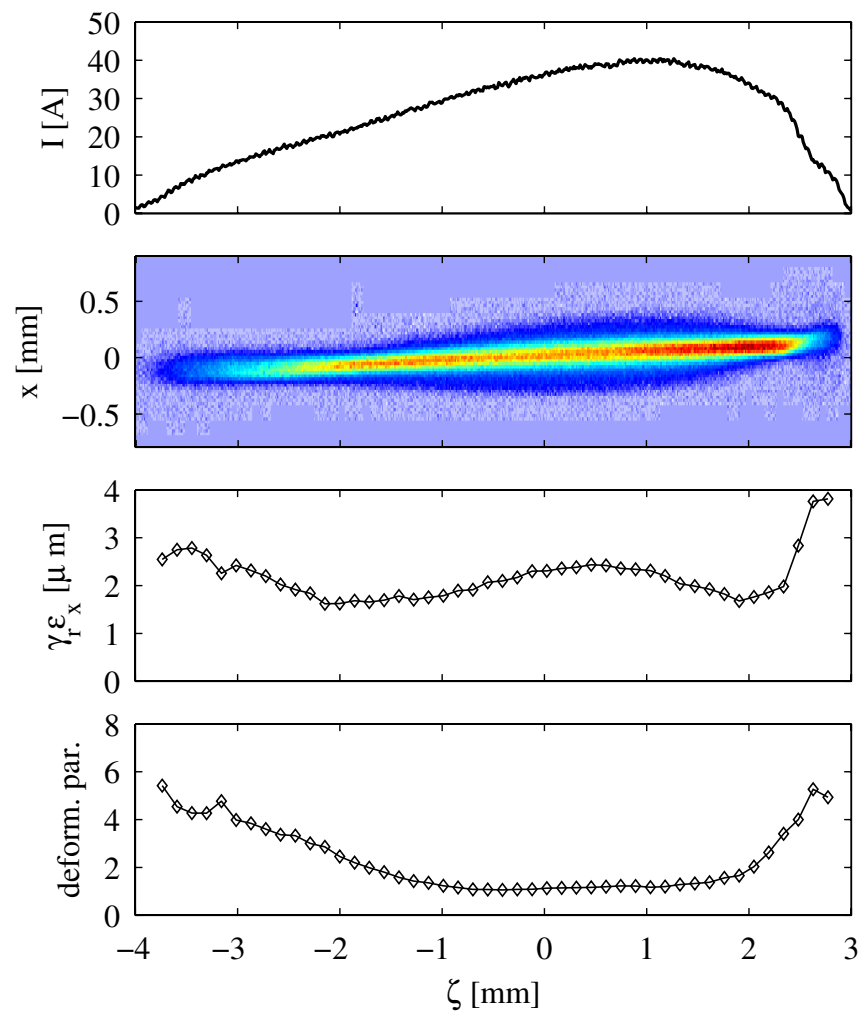

FIG. 9. (Color) Experimental results for bunches accelerated oncrest in all acceleration modules ( $E=490 \mathrm{MeV}, Q=0.6 \mathrm{nC})$. From top to bottom: Average current profile, typical CCD image, normalized slice emittance, and deformation parameter (see text). The bunch compressor magnets were switched on. Statistical errors of slice emittance and deformation parameter are in the order of a few percent and are not shown here. the range of 1.6 to $4 \mu \mathrm{m}$. In the center region values around $2 \mu \mathrm{m}$ are observed, while at the head and tail of the bunch the slice emittance increases. The phase space ellipses of slices in these bunch regions are misaligned with respect to those in the bunch center and to the phase space ellipse of the entire bunch. In order to quantify this effect, we introduce a "deformation parameter"

$$
\begin{aligned}
m(\zeta) & =B(\zeta)+\sqrt{B(\zeta)^{2}-1} \\
\text { with } B(\zeta) & =\frac{1}{2}\left[\beta(\zeta) \gamma^{0}+\gamma(\zeta) \beta^{0}-2 \alpha(\zeta) \alpha^{0}\right] .
\end{aligned}
$$

Here, $\left(\beta^{0}, \alpha^{0}, \gamma^{0}\right)$ are the averaged beam ellipse parameters of the entire bunch and $[\beta(\zeta), \alpha(\zeta), \gamma(\zeta)]$ those within a slice at position $\zeta$. The definition (15) is the generalization of the well-known betatron mismatch parameter [32] and describes the misalignment of two beam ellipses with respect to each other. As shown in Fig. 9, the growth in slice emittance at both ends is accompanied by an increasing deformation parameter up to $m \approx 5$. The origin of these deformations is space charge forces in the electron gun. The focusing solenoid is adjusted to compensate for the transverse space charge force in the center of the bunch. As a consequence, the space charge forces remain partly uncompensated in the low-current regions in the head and the tail.

A tomographic reconstruction of the horizontal phase space distribution of a single slice in the bunch center is shown in Fig. 10(a). The distribution has an elliptical shape and can be approximated well by a two-dimensional Gaussian density distribution. The normalized emittance is $2.0 \mu \mathrm{m}$. This value agrees within $10 \%$ with the one obtained using a least squares method, showing that the reconstruction is quite accurate.

In contrast to the case of a single slice, the reconstructed phase space distribution of the entire bunch [Fig. 10(b)] shows significant deviations from a Gaussian distribution and covers a larger area in phase space. Consequently, the overall emittance, often called the "projected" emittance, amounts to $3.8 \mu \mathrm{m}$ and is thus nearly twice as large as the average, current-weighted slice emittance of $2.1 \mu \mathrm{m}$. The large value of the projected emittance has two reasons:
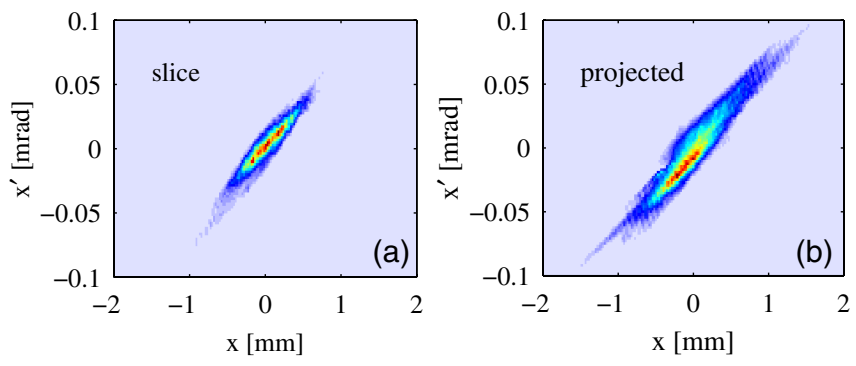

FIG. 10. (Color) Tomographic reconstruction of the horizontal phase space distribution of (a) a single slice at $\zeta=0$ and (b) an entire uncompressed bunch. 


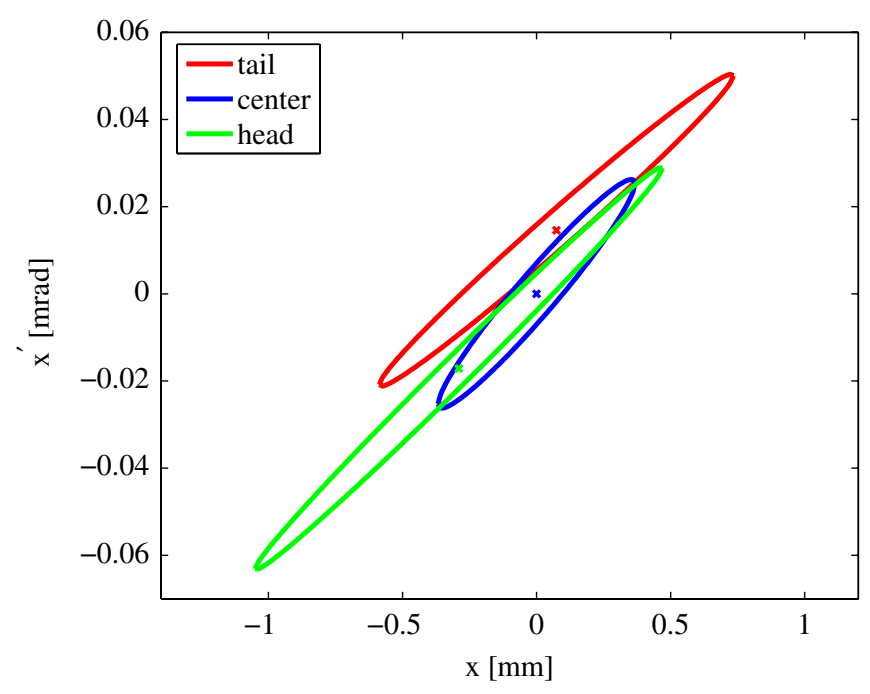

FIG. 11. (Color) Phase space ellipses of single slices in the tail, center, and head of an uncompressed bunch. The coordinates $\left[\langle x(\zeta)\rangle,\left\langle x^{\prime}(\zeta)\right\rangle\right]$ of the slice centroids are indicated by $\mathrm{x}$ marks.

misalignments of the slice ellipses with respect to each other, and a $\zeta$ dependence of the slice centroid coordinates $\langle x(\zeta)\rangle$ and $\left\langle x^{\prime}(\zeta)\right\rangle$, see Fig. 11. Eliminating the contribution from centroid shifts, the projected emittance drops to $2.5 \mu \mathrm{m}$, showing that centroid shifts are the dominant source for the observed projected emittance growth.

\section{EXPERIMENTAL RESULTS UNDER FEL OPERATING CONDITIONS}

Prior to the measurements of longitudinally compressed bunches, the machine was tuned for SASE operation in the exponential gain regime, yielding FEL pulse energies of 5-10 $\mu \mathrm{J}$. During the diagnostic measurements, the strengths of several quadrupoles in the diagnostic beam line had to be varied. The resulting change in beam orbit and beam size in the undulator magnets led to an interruption of the SASE FEL process. The variation of the optics in the diagnostic beam line has practically no influence on fundamental beam properties such as the transverse slice emittance and the longitudinal phase space distribution. Therefore, the results presented in the following sections should be representative for the FEL operation of FLASH. The TDS was operated with a large shear parameter $S=20$ in order to achieve optimum time resolution, at the expense of a restricted time window.

\section{A. Current profile}

Figure 12(a) shows the average current profile measured under FEL operating conditions at a bunch charge of $0.5 \mathrm{nC}$ and an electron energy of $677 \mathrm{MeV}$. The bunch profile is characterized by a sharp leading spike and a long tail. The spike contains the part of the bunch which pro-
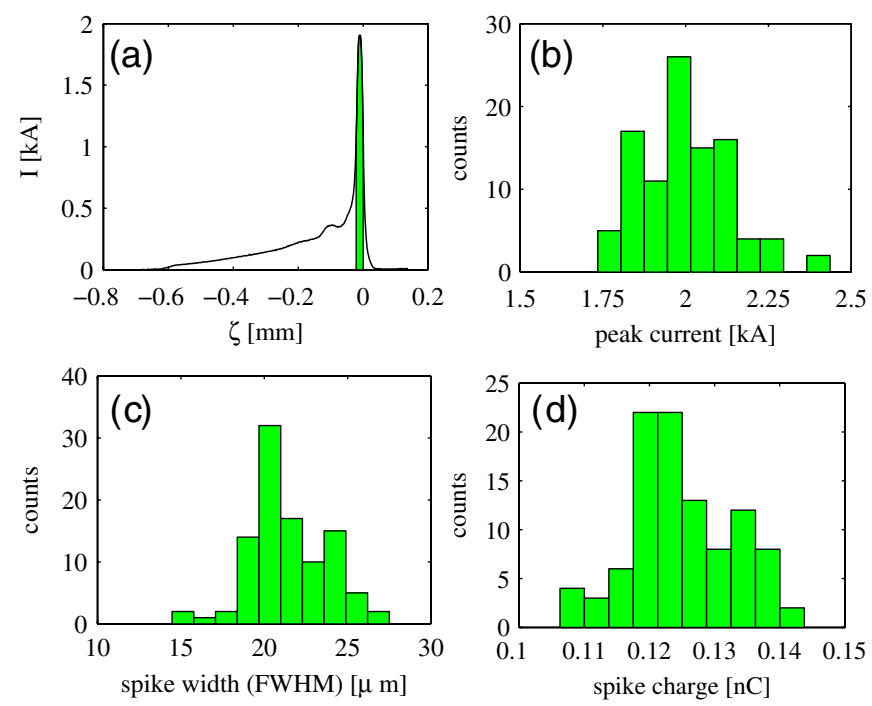

FIG. 12. (Color) (a) Average current profile of a compressed bunch $(E=677 \mathrm{MeV}, Q=0.5 \mathrm{nC})$. The region of the current spike is indicated by the colored area. (b) Histogram of the peak current distribution of 100 subsequent bunches, the average value is $I_{\text {peak }}=(2.0 \pm 0.1) \mathrm{kA}$. (c) and (d) Corresponding histograms for the width and the charge of the colored region in (a). The mean width of the spike is (22 \pm 3$) \mu \mathrm{m}$ (FWHM), the mean charge is $(0.13 \pm 0.01) \mathrm{nC}$.

duces the FEL radiation and is thus of particular interest. The measured peak current is $(2.0 \pm 0.1) \mathrm{kA}$, and the spike width (FWHM) is $(22 \pm 3) \mu \mathrm{m}$. Note that the internal bunch coordinate $\zeta$ is defined here with respect to the half-maximum position of the sharp leading edge of the current spike instead of the center of mass of the bunch.

The charge fraction contained in the spike is about $25 \%$. Measurements at other electron energies yielded a lower charge fraction in the spike and a longer tail. A small accumulation of charge can be observed in front of the spike. This is a typical indication for the action of longitudinal space charge forces in the linac section between the two bunch compressors, by which the electrons at the very front gain energy and travel on a shorter path through $\mathrm{BC} 2$ [33].

An important issue for FEL operation is the stability of the machine on a shot-to-shot basis. As shown in Figs. 12(b)-12(d), the peak current, the width of the current spike, and the charge inside the spike fluctuate by only $7 \%$ to $12 \%$ (rms) around their mean value. Variations of the bunch profile from shot to shot are mainly due to fluctuations of the rf phase of the electron gun and the first acceleration module ACC1. These phase variations are typically less than $0.1^{\circ}$ (rms) [34].

The estimated longitudinal rms resolution of the profile measurements is $8 \mu \mathrm{m}$ (19 $\mu \mathrm{m}$ FWHM). Because of this limitation, the true peak current is expected to be larger and the spike width smaller than the quoted values. 


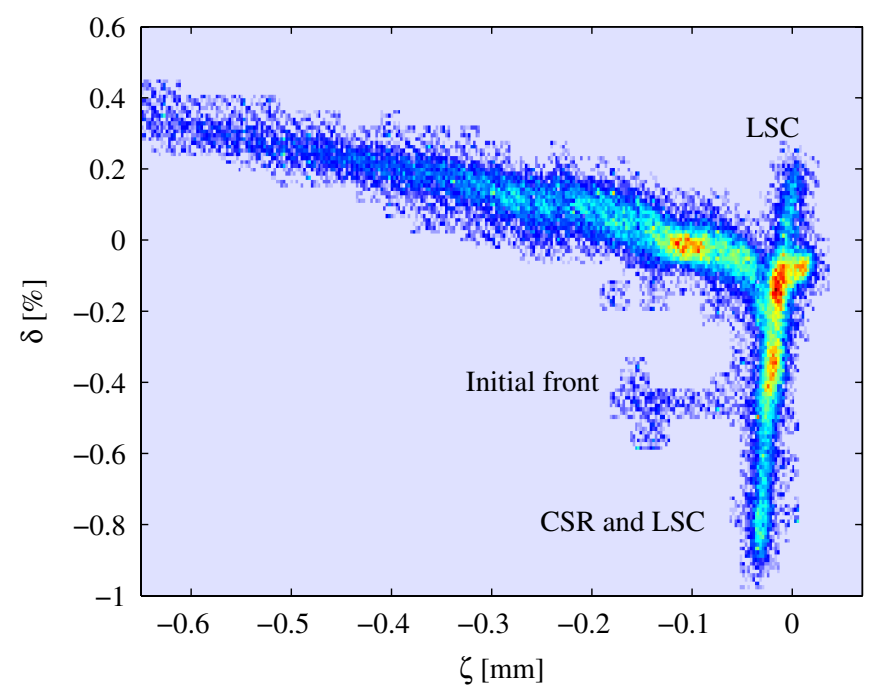

FIG. 13. (Color) Single-shot measurement of the charge density distribution in longitudinal phase space at $677 \mathrm{MeV}$. The vertical axis shows the relative momentum deviation $\delta$ and the horizontal axis the internal longitudinal position $\zeta$. Abbreviations: longitudinal space charge (LSC), coherent synchrotron radiation (CSR).

\section{B. Longitudinal phase space}

The CCD image in Fig. 13 shows a single-shot measurement of the energy-dispersed charge density distribution on the screen OTR2 (cf. Fig. 1). Again, most of the bunch charge is spread out over a long tail which possesses an energy gradient resulting from the off-crest acceleration. Within the current spike, the energy-dependent path length in the magnetic chicanes causes bunch fractions with different energies to overlap at the same longitudinal position. Particles that were initially at the very front of the bunch have gained the least energy in the acceleration modules and are now trailing behind the high-current region.

The energy profile of the current spike is strongly broadened due to collective effects. Numerical simulations $[28,33]$ show that the observed structures can be attributed to longitudinal space charge forces (LSC) and coherent synchrotron radiation (CSR) in the bunch compressor chicanes.

The rms slice energy spread in the tail amounts to $\sigma_{E} \approx$ $340 \mathrm{keV}\left(\sigma_{\delta} \approx 0.05 \%\right)$. This value is almost 2 orders of magnitude larger than predicted by numerical simulations and is hence regarded as an estimate for the energy resolution of the beam optical setup as used for the investigation of compressed bunches. The slice energy spread of the current spike is found to be about $1.7 \mathrm{MeV}(0.25 \%)$, which is clearly above the estimated resolution of $340 \mathrm{keV}$. In contrast to this, FEL theory and numerical beam simulations predict an energy spread of the lasing fraction of less than $0.1 \%$ or $680 \mathrm{keV}$ [35]. From this obvious discrepancy, we conclude that only a fraction of the electrons within the current spike is able to contribute to the lasing process.
This assumption is corroborated by the horizontal phase space analysis presented in the following section.

\section{Horizontal phase space}

The transverse emittance of the electron beam is a crucial parameter for the gain length in a SASE FEL (see e.g. [35]). From the FEL gain measured at FLASH, one deduces a normalized transverse emittance of a few micrometers [8]. However, a projected horizontal bunch emittance of more than $10 \mu \mathrm{m}$ was repeatedly measured under FEL operating conditions. To clarify the situation, a tomographic analysis was carried out. Figure 14 shows the reconstructed horizontal phase space distribution at $494 \mathrm{MeV}$. The distribution exhibits strong deviations from the ideal elliptic shape and is not centered at the origin $\left(x, x^{\prime}\right)=(0,0)$. Instead, it features two wellseparated intensity maxima, a strong one at $\langle x\rangle \approx$ $0.5 \mathrm{~mm},\left\langle x^{\prime}\right\rangle \approx 0.04 \mathrm{mrad}$, and a weaker one at $\langle x\rangle \approx$ $-1.2 \mathrm{~mm},\left\langle x^{\prime}\right\rangle \approx-0.1 \mathrm{mrad}$. The large separation between the intensity maxima explains the high value of the projected emittance of $13 \mu \mathrm{m}$.

Making use of the longitudinal information provided by the TDS we have determined the charge centroid (center of the charge density) in the $\left(x, x^{\prime}\right)$ plane for each longitudinal slice of the bunch by computing $\left[\langle x(\zeta)\rangle,\left\langle x^{\prime}(\zeta)\right\rangle\right]$. The three-dimensional representation of the slice centroid curve is depicted in Fig. 15. One observes strong deviations from a straight line parallel to the $\zeta$ axis that would be expected in case linear beam optics were valid. A small deviation from such a straight line behavior will occur due to the horizontal dispersion of the kicker magnet of $10 \mathrm{~mm}$ and some spurious dispersion in the beam line, but the major source is self-field effects of the bunch: coherent

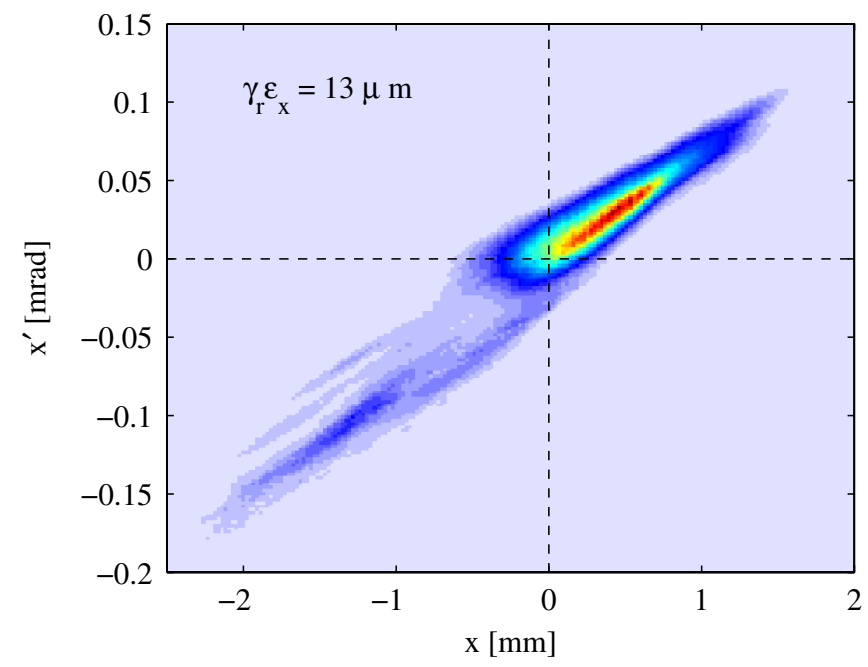

FIG. 14. (Color) Tomographic reconstruction of the horizontal phase space distribution of a compressed bunch, averaged over the entire bunch length. The intersection of the dashed lines defines the charge centroid (energy $E=494 \mathrm{MeV}$, bunch charge $Q=0.72 \mathrm{nC})$. 


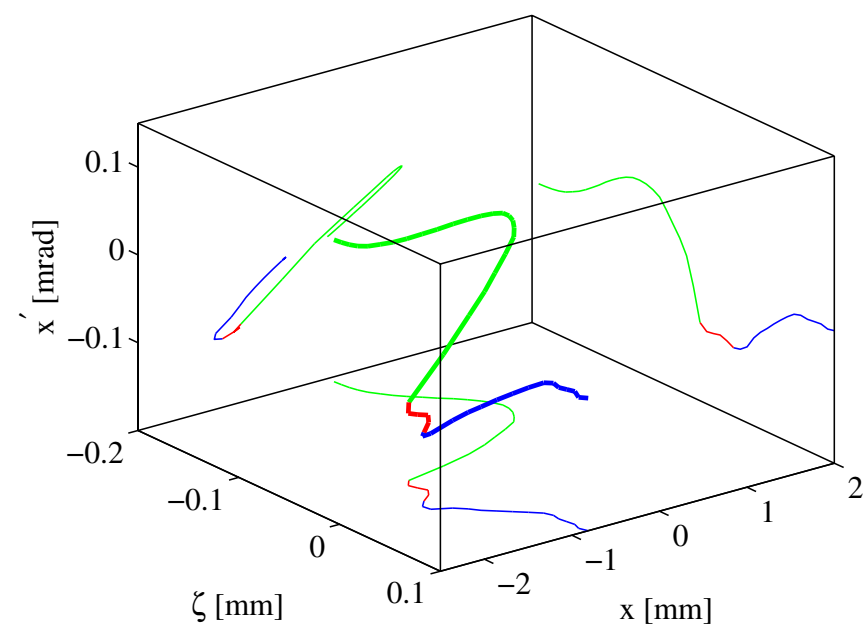

FIG. 15. (Color) Three-dimensional representation of the slice centroid as a function of the longitudinal coordinate $\zeta$ (bold curve). Green: bunch tail; red: current spike; blue: electrons in front of the current spike. The thin curves show the projections of the centroid curve onto the $\left(x, x^{\prime}\right),(x, \zeta)$, and $\left(x^{\prime}, \zeta\right)$ planes, respectively. Note that the bunch tail is truncated at $\zeta=$ $-0.2 \mathrm{~mm}$.

synchrotron radiation, wake fields, and space charge forces.

The centroid curve reveals that the strong intensity maximum in Fig. 14 can be attributed to the bunch tail, while the weaker maximum is generated by the leading current spike. Beam dynamics simulations [28] suggest that the current spike experiences a horizontal displacement in the second bunch compressor chicane mainly due to energy losses by coherent synchrotron radiation.

The slice emittance is depicted in Fig. 16. Within the bunch tail, the emittance has values of around $2 \mu \mathrm{m}$, but it becomes surprisingly large in the region of the current spike. The maximum value of $13 \mu \mathrm{m}$ occurs close to the trailing edge of the current spike, where a large horizontal width and an asymmetry can be observed in the recorded camera images. Within the spike, the slice emittance is in the range of 7.5 to $12 \mu \mathrm{m}$, with a local minimum of $7.5 \mu \mathrm{m}$ close to the leading edge. The region with an increased slice emittance is significantly longer than the current spike. The bunch fraction in front of the spike has an emittance of 3 to $9 \mu \mathrm{m}$. The deformation parameter (compare Sec. IV C) is moderate up to the rear edge of the leading spike and then continuously grows up to $m \approx 5$. The longitudinal resolution of the measurement is about $8 \mu \mathrm{m}$ (rms).

Figure 17 shows the reconstructed horizontal phase space distributions of selected slices along the bunch. Within the current spike ( $-32 \mu \mathrm{m} \leq \zeta \leq 0)$, a bifurcation into two "branches" is observed, which is a major contribution to the enhanced slice emittance. Furthermore, a high-intensity core can be seen in the center of the distributions throughout the current spike. The total phase
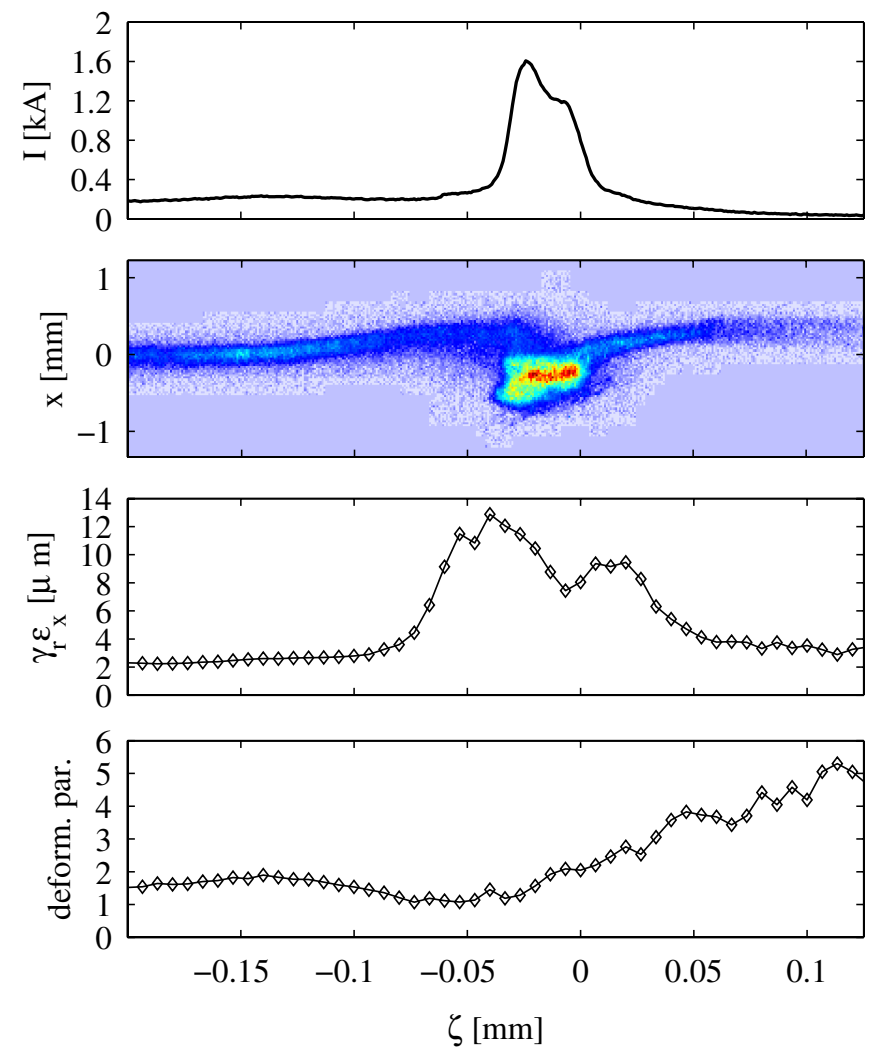

FIG. 16. (Color) Experimental results under FEL operating conditions at $494 \mathrm{MeV}$. From top to bottom: Average current profile, typical CCD image, normalized slice emittance, and deformation parameter. A large shear parameter $S=29$ has been chosen to achieve good resolution, hence only a limited section of the bunch is visible on the screen and most of the bunch tail is truncated.

space area covered by the electrons in the bunch head is significantly larger than in the tail $(\zeta=-107 \mu \mathrm{m})$.

The slice emittance in the region of the current spike is far larger than the value of a few $\mu \mathrm{m}$ deduced from the measured FEL gain length [8]. The phase space distribution of a slice within the current spike is depicted in Fig. 18(a) and 18(b). The observation of a high-intensity core in horizontal phase space suggests that only a fraction of the particles in the current spike with a much smaller emittance contributes to the lasing process. In order to quantify the emittance of the high-intensity core, a twodimensional Gaussian fit was performed after cutting the low-intensity regions of the slice distributions by applying a threshold procedure using the half-maximum intensity. The Gaussian distribution obtained in this way has indeed a small emittance of 2-3 $\mu \mathrm{m}$ [Figs. 18(c) and 18(d)]. Most importantly, the current of $0.6-0.8 \mathrm{kA}$ contained in the core is sufficiently high to drive the FEL gain process. According to "start-to-end" simulations [36], the peak current is expected to grow by about $25 \%$ when the bunch passes the double-bend magnetic deflection (the "dog leg") in the collimator region (see Fig. 1), which is bene- 


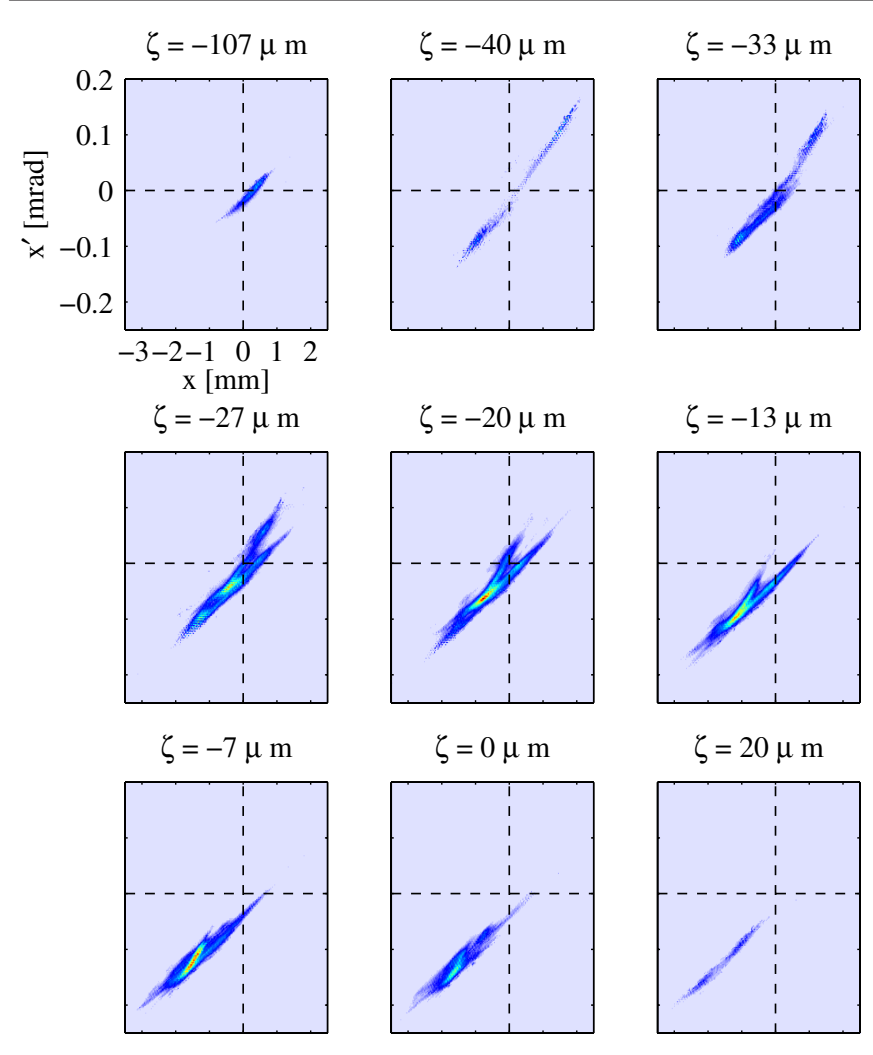

FIG. 17. (Color) Reconstructed horizontal phase space distributions of selected slices. The longitudinal position of the individual slices is indicated on top of the images. The charge density scale is the same in each image. Note that the origin $(0,0)$ is defined by the centroid of the charge distribution of the entire bunch, compare Fig. 14.

ficial for the FEL gain. A core emittance of a few $\mu \mathrm{m}$ and a peak current in the order of $1 \mathrm{kA}$ were also found at electron energies of 677 and $964 \mathrm{MeV}$ [28].
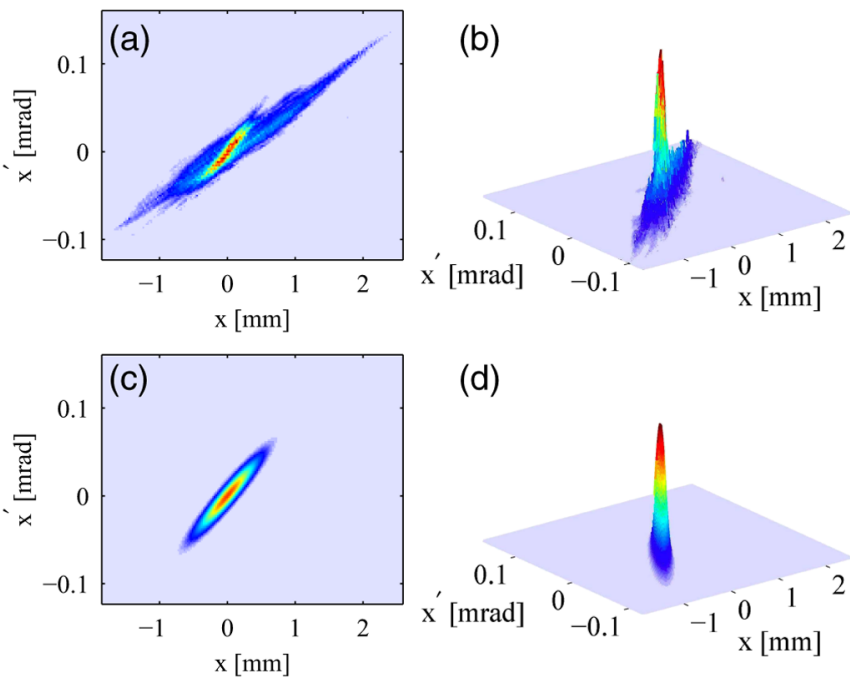

(d)

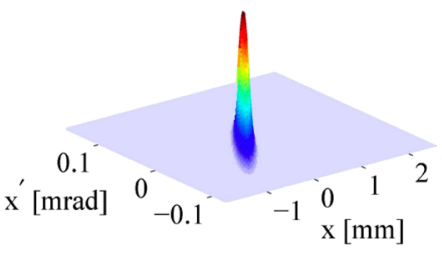

FIG. 18. (Color) (a) and (b): Measured distribution of a slice within the current spike at $494 \mathrm{MeV}(\zeta=-7 \mu \mathrm{m})$. (c) and (d): Two-dimensional Gaussian fit to the high-intensity core.

\section{SUMMARY}

We have carried out the first time-resolved analysis of the horizontal and longitudinal phase space distribution of electron bunches in a high-gain free-electron laser, using a transverse deflecting microwave structure with a time resolution of 27 fs under FEL operating conditions. The leading spike of the compressed electron bunches was measured to have a peak current of up to $2 \mathrm{kA}$, a length (FWHM) of 20 to $30 \mu \mathrm{m}$ (67 to $100 \mathrm{fs}$ ), an rms slice energy spread of up to $1.7 \mathrm{MeV}$, and a horizontal slice emittance in the range of 8 to $12 \mu \mathrm{m}$. Since energy spread and transverse emittance are far higher than expected from the measured FEL gain [8], we conclude that only a fraction of the electrons in the high-current region contribute to the FEL process. By applying a time-resolved transverse phase space tomography, the existence of a central core with low emittance and high peak current could be demonstrated. This core is presumably driving the SASE FEL process.

When comparing the experimental results obtained under FEL operating conditions to FEL theory and numerical simulations, it must be kept in mind that the experiments took place upstream of the dispersive collimator section (cf. Fig. 1), in which a slight modification of the bunch shape and a 25\% increase in peak current are to be expected. Moreover, there are principle limitations of the TDS technique. The longitudinal resolution of about $8 \mu \mathrm{m}$ is large compared to the coherence length of the FEL process at FLASH, which is on the order of $1 \mu \mathrm{m}$ [8]. Resolution limitations lead to an apparent reduction of the peak current and also affect the slice emittance. Furthermore, the tomographic reconstruction refers to the average phase space distribution of typically 200 electron bunches. Shot to shot fluctuations generally lead to a dilution of the average phase space distribution. The measured emittance is thus an upper limit for the true singlebunch emittance.

In summary, the time-resolved phase space tomographic technique has turned out to be essential for an understanding of the complicated transverse phase space structure of the longitudinally compressed electron bunches in a highgain free-electron laser. A detailed comparison of the experimental results with numerical simulations is in progress. The main difficulty of such a comparison arises from the high sensitivity of beam properties to machine parameters, in particular, to the rf phases of the acceleration modules in FLASH, which are presently not known with sufficient accuracy.

\section{ACKNOWLEDGMENTS}

We are very grateful to J. Frisch, D. McCormick, M. Ross, T. Smith, A. Bolzmann, M. Hüning, R. Jonas, M. Nagl, and J. Rossbach of the SLAC-DESY team for the installation and commissioning of the TDS in the FLASH 
linac. We thank the FLASH team for support.

[1] W. E. Stein and R. L. Sheffield, Nucl. Instrum. Methods Phys. Res., Sect. A 250, 12 (1986).

[2] O. H. Altenmueller, R. Larsen, and G. A. Loew, Rev. Sci. Instrum. 35, 438 (1964).

[3] P. Emma, J. Frisch, and P. Krejcik, Technical Report No. LCLS-TN-00-12, 2000.

[4] R. Akre et al., Phys. Rev. ST Accel. Beams 11, 030703 (2008).

[5] R. Brinkmann, K. Flöttmann, J. Rossbach, P. Schmüser, N. Walker, and H. Weise, Technical Design Report Part 2, No. ECFA-2001-209, 2001.

[6] V. Ayvazian et al., Phys. Rev. Lett. 88, 104802 (2002).

[7] V. Ayvazian et al., Eur. Phys. J. D 20, 149 (2002).

[8] W. Ackermann et al., Nat. Photon. 1, 336 (2007).

[9] S. Schreiber, B. Faatz, and K. Honkavaara, in Proceedings of the 11th European Particle Accelerator Conference, Genoa, 2008 (EPS-AG, Genoa, Italy, 2008), MOPC030.

[10] M. Altarelli et al., Technical Report No. DESY-06-097, 2006.

[11] J. Brau et al., Report No. ILC-REPORT-2007-001, 2007.

[12] A. Abrahamyan et al., Nucl. Instrum. Methods Phys. Res., Sect. A 528, 360 (2004).

[13] B. Aune et al., Phys. Rev. ST Accel. Beams 3, 092001 (2000).

[14] A. Bolzmann, Diploma thesis, University of Hamburg [DESY-THESIS-2005-046, 2005].

[15] K. Honkavaara et al., in Proceedings of the 20th Particle Accelerator Conference, Portland, OR, 2003 (IEEE, New York, 2003), p. 2476.

[16] M. Hüning et al., in Proceedings of the 27th International Free Electron Laser Conference, Stanford, California, 2005, THPP035.

[17] H. Loos et al., Report No. SLAC-PUB-13395, 2008.

[18] B. Beutner, Contribution to the Microbunching Instability Workshop, LBNL, Berkeley, 2008, http://www. elettra.trieste.it/FERMI/index.php?

$\mathrm{n}=$ Main.MicrobUSScientProgr.
[19] G. Berden et al., Phys. Rev. Lett. 99, 164801 (2007).

[20] S. Casalbuoni et al., Phys. Rev. ST Accel. Beams 12, 030705 (2009).

[21] B. Schmidt et al., prepared for the 30th International Free Electron Laser Conference, Gyeongju, Korea, 2008, TUPPH068; contribution to the Microbunching Instability Workshop, LBNL, Berkeley, 2008, http:// www.elettra.trieste.it/FERMI/index.php? $\mathrm{n}=$ Main.MicrobUSScientProgr.

[22] M. G. Minty and F. Zimmermann, Measurement and Control of Charged Particle Beams (Springer, Berlin, 2003).

[23] O. R. Sander, G. N. Minerbo, R. A. Jameson, and D. D. Chamberlin, in Proceedings of the Linear Accelerator Conference, Montauk, New York, 1979, p. 314.

[24] G. N. Minerbo, O. R. Sander, and R. A. Jameson, IEEE Trans. Nucl. Sci. 28, 2231 (1981).

[25] G. Minerbo, Computer Graphics Image Process. 10, 48 (1979).

[26] J. Scheins, Report No. DESY-TESLA-2004-08, 2004.

[27] V. Yakimenko, M. Babzien, I. Ben-Zvi, R. Malone, and X. J. Wang, Phys. Rev. ST Accel. Beams 6, 122801 (2003).

[28] M. Röhrs, Ph.D. thesis, University of Hamburg [Report No. DESY-THESIS-2008-012, 2008].

[29] M. Dohlus et al., Nucl. Instrum. Methods Phys. Res., Sect. A 530, 217 (2004).

[30] K. Flöttmann, http://www.desy.de/ mpyflo (2000).

[31] M. Hüning and H. Schlarb, in Proceedings of the 20th Particle Accelerator Conference, Portland, OR, 2003 (IEEE, New York, 2003), p. 2074.

[32] M. Sands, Report No. SLAC-AP-085, 1991.

[33] M. Dohlus, in Proceedings of the 10th European Particle Accelerator Conference, Edinburgh, Scotland, 2006 (EPSAG, Edinburgh, Scotland, 2006), p. 1847.

[34] H. Schlarb et al., in Proceedings of the 10th European Particle Accelerator Conference, Edinburgh, Scotland, 2006 (Ref. [33]), p. 1052.

[35] P. Schmüser, M. Dohlus, and J. Rossbach, Ultraviolet and Soft X-Ray Free-Elecron Lasers (Springer, Berlin, 2008).

[36] M. Dohlus, EUROFEL Workshop, Saclay, France, 2005. 